\title{
Spontaneous heterotopic pregnancy with tubal rupture
}

\section{Spandana J. C.*, Suresh S. Kanakannavar, Umashankar K. M., Manuja N.}

Department of Obstetrics and Gynecology, BMCRI, Bangalore, Karnataka, India

Received: 23 August 2017

Accepted: 16 September 2017

\section{*Correspondence: \\ Dr. Spandana J. C., \\ E-mail:drspanjc@gmail.com}

Copyright: () the author(s), publisher and licensee Medip Academy. This is an open-access article distributed under the terms of the Creative Commons Attribution Non-Commercial License, which permits unrestricted non-commercial use, distribution, and reproduction in any medium, provided the original work is properly cited.

\begin{abstract}
Heterotopic pregnancy is the coexistence of intrauterine pregnancy (IUP) and extrauterine gestation. It is a rare and dangerous life-threatening condition that is difficult to diagnose and easily missed. The incidence in the general population is estimated to be 1 in 30,000 . We report a case of a 24 -year-old multigravida who was seen in the emergency department with a diagnosis of a ruptured ectopic pregnancy. A careful ultrasound assessment led to the diagnosis of a heterotopic pregnancy despite lack of any notable risk factors. Immediate surgical intervention with supportive measures resulted in a successful outcome. An obstetrician should keep in mind the occurrence of a heterotopic pregnancy while dealing with pregnant females. It also demonstrates that early diagnosis is essential in order to salvage the intrauterine pregnancy and avoid maternal morbidity and mortality.
\end{abstract}

Keywords: Ectopic pregnancy, Heterotopic pregnancy, Ultrasonography

\section{INTRODUCTION}

Heterotopic pregnancy(HP) is the coexistence of intrauterine pregnancy(IUP) and extrauterine gestation. ${ }^{1}$ It is a rare and dangerous life threatening condition that is difficult to diagnose and easily missed. ${ }^{2}$ The incidence in the general population is estimated to be 1 in 30,000 natural conception while a rate as high as 1 in 8,000 has been reported. ${ }^{3}$ The fallopian tube is the common site of the majority of the ectopic implantation in heterotopic pregnancies, but the cervix or abdomen can also be involved. ${ }^{4}$

Risk factors for ectopic pregnancy are pelvic inflammatory disease (PID), tubo-ovarian abscess (TOA), previous surgery or previous ectopic pregnancies. ${ }^{5}$ Heterotopic pregnancy is thought to occur because of multiple ovulation events. Therefore, individuals who have undergone assisted reproduction therapies are at an increased risk of $\mathrm{HP}^{3}$ Pregnancies with assisted reproduction have an estimated $1 \%$ chance of culminating in a heterotopic pregnancy so it should be suspected in any patient who presents with lower abdominal pain in a pregnancy resulting from fertility treatment. ${ }^{6}$

Individuals often present with symptoms like abdominal mass, abdominal pain, peritoneal irritation, and enlarged uterus $^{3}$, although in some cases there may be either hypovolemic shock or a complete lack of symptoms. ${ }^{7}$ Early symptoms can also be similar to those seen in acute appendicitis, ovarian cyst rupture, or ovarian torsion, further adding to difficulty in diagnosis. ${ }^{7}$

Diagnosis is challenging, as it is often difficult to identify both the IUP and extrauterine pregnancy. A significant discrepancy between beta human chorionic gonadotropin levels and the corresponding IUP can reinforce the possibility of another pregnancy. ${ }^{7}$ Approximately $70 \%$ of heterotopic pregnancies are diagnosed between 5-8 weeks, $20 \%$ are diagnosed between weeks 9 and 10, and the remaining $10 \%$ are diagnosed at or beyond the 11 th week. ${ }^{8}$ We report a case of HP in a natural conception cycle that presented with tubal rupture. 


\section{CASE REPORT}

A 24 year old gravida 3 para 2 living 2 was referred to our centre, Bowring and Lady Curzon Hospital attached to Bangalore Medical college. She presented to the emergency Department with history of two and half months of amenorrhea and acute crampy lower abdomen pain since 6 hours and brief episode of loss of consciousness. She was 10 weeks pregnant. It was a spontaneous conception and there was no past history of abortion, infertility treatment, pelvic inflammatory disease or any history of abdominal surgery.

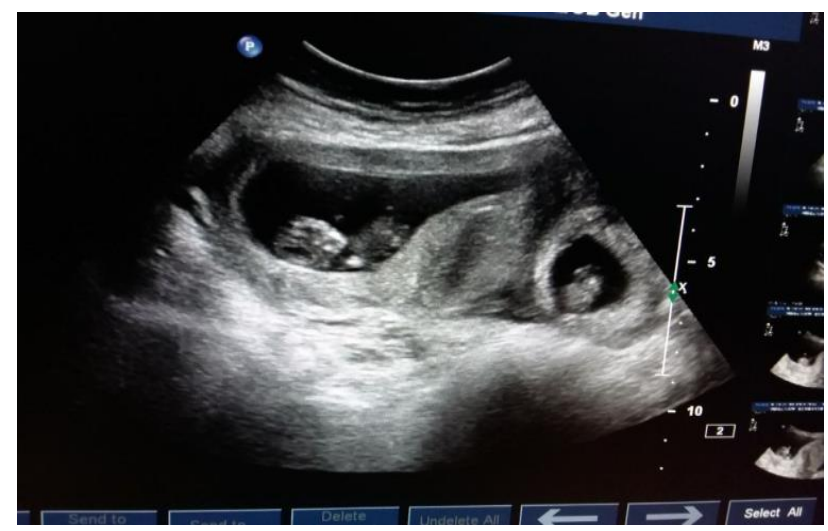

Figure 1: Transabdominal ultrasound image of the heterotopic pregnancy showing viable pregnancies in both the left adnexa and uterus.

On examination, she was pale with a pulse rate of 96 per minute and blood pressure of $100 / 70 \mathrm{mmHg}$. Abdominal examination revealed diffuse, lower abdominal tenderness. Bimanual examination revealed an anteverted, enlarged, soft uterus corresponding to 10 weeks of pregnancy.

In addition, a tender mass was also palpable in her left adnexa. Cervical movements were painful but there was no bleeding.

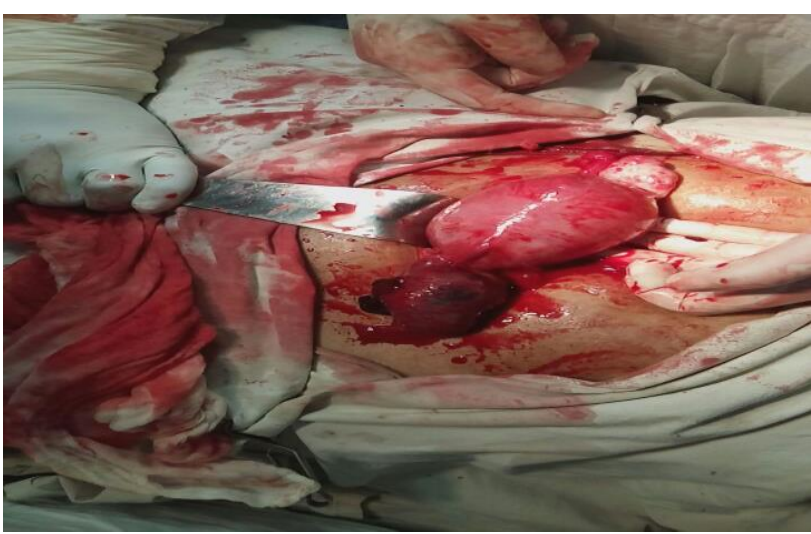

Figure 2: Operative findings during emergency laparotomy showing a ruptured left tubal pregnancy in the ampullary area and an increased globular uterus with hemoperitoneum.
Her urine pregnancy test was positive. Her serum beta HCG was 1,53,000 $\mathrm{mIU} / \mathrm{mL}$. Urine dipstick showed no signs of uri $\neg$ nary tract infection. Her haemoglobin was $8.9 \mathrm{~g} / \mathrm{dL}$.

She underwent a transabdominal and transvaginal ultra $\neg$ sound scan which revealed a live intrauterine gesta $\neg$ tion of 10 weeks. In addition, however there was a left adnexal mass with a gestational sac and a fetal pole suggestive of an ectopic pregnancy as shown in figure 1 . There was a faint fetal heart seen in the fetal pole. A moderate amount of fluid was present in the cul-de-sac and a diagnosis of heterotopic pregnancy with tubal rupture was made.

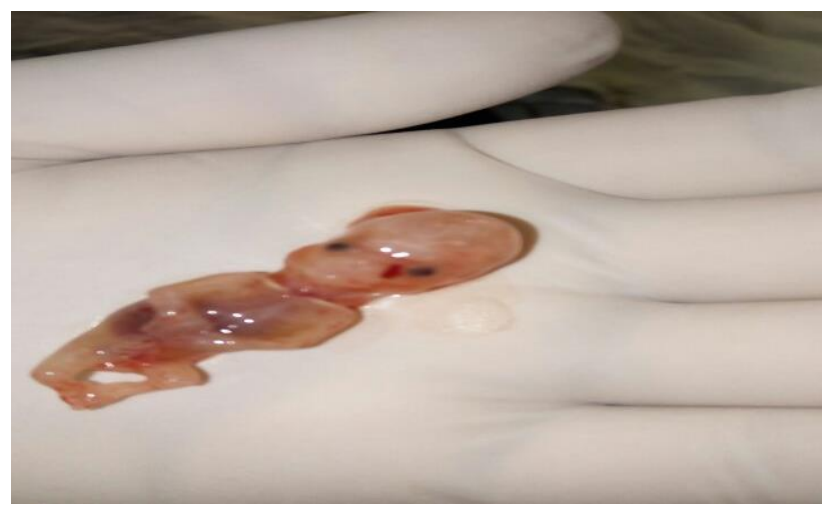

Figure 3: Operative findings after emergency laparotomy showing the extra-uterine embryo.

An emergency laparotomy on the patient revealed 10 week gravid uterus and rupture of the left tube near the ampullary end and the presence of approximately $700 \mathrm{ml}$ of hemoperitoneum as shown in figure 2 . Left salpingectomy with evacuation of clots and products was done and peritoneal lavage was performed. A pelvic ultrasound scan confirmed a viable 10 weeks intrauterine pregnancy. Histology of the salpingectomy specimen confirmed an ectopic pregnancy. Postoperative period was uneventful and was discharged on post op day 7. She opted to continue with the intrauterine pregnancy.

\section{DISCUSSION}

HP is diagnosed in the presence of multiple pregnancies with one or more intrauterine pregnancies co-existing with an ectopic pregnancy. ${ }^{1}$ The ectopic pregnancy can be tubal, ovarian, cervical, cornual or abdominal. Tubal ectopic pregnancies are the most common. The occurrence of a heterotopic pregnancy is considered rare in natural conception cycles with an incidence of $0.08 \%$, but incidence increases to as high as $1-2.9 \%$ with assisted reproductive techniques. ${ }^{6}$

There are two categories of risk factors for heterotopic pregnancy: risk factors of ectopic pregnancy (history of infertility, sexually transmitted infection, intrauterine device, smoking, hormonal contraception, pelvic surgery) and ovulation induction and assisted reproductive 
technologies (ART). ${ }^{5}$ Our patient did not have any risk factor of ectopic pregnancy.

This case represents a spontaneous heterotopic pregnancy in a 24-year-old patient with no previous risk factors identified.

Early diagnosis of heterotopic pregnancy may not be easy in our set up where first trimester and emergency room pelvic ultrasonography are not routinely performed. In those situation the most probable diagnosis will be that of a ruptured ectopic pregnancy leading to inappropriate handling of the uterus during surgery with adverse effects on the intrauterine foetus.

It illustrates the importance of not immediately ruling out an IUP after identifying an ectopic pregnancy, and consistently placing a heterotopic pregnancy on the differential diagnosis. In the case of confirmed IUP and hemoperitoneum, it is important to consider the possibility of ruptured heterotopic pregnancy, as was seen in the case presented here.

Serial b-HCG levels are not of much significance in the diagnosis of HP as subnormal hormone production by an ectopic pregnancy may be masked by the higher placental production from the intrauterine pregnancy. Culdocentesis is an important aid in diagnosis when hemoperitoneum is present as echogenic pelvic fluid is more important than anechoic fluid because it indicates the presence of peritoneal haemorrhage. ${ }^{9}$

In the case of ectopic pregnancy undergoing surgical management, intrauterine device such as uterine manipulator should be generally avoided due to the likelihood of coexistence of early intrauterine pregnancy that is not visualized by ultrasound.

Fortunately, in this case, the IUP and extrauterine pregnancy were discovered simultaneously via ultrasound. Conservative surgical management will allow the viable IUP to develop to term.

In up to $50 \%$ of cases, heterotopic pregnancy can remain totally asymptomatic till it is discovered by a routine first trimester ultrasound. ${ }^{10}$ The treatment of heterotopic pregnancy can be surgical, medical or expectant. Out of 217 cases of heterotopic pregnancies reported in literature, $90.78 \%$ were managed surgically. ${ }^{11}$ The procedure usually consists of salpingectomy via laparotomy or laparoscopy depending on patient's hemodynamic state at the time of diagnosis. Depending on the state of the controlateral fallopian tube, on the patient's reproductive needs, and on the state of the ectopic pregnancy it is possible to consider conservation of the tube hosting the pregnancy.

In present case, emergency laparotomy was carried out due to lack of equipment for laparoscopy and we did a total left salpingectomy because of important tubal damage. Heterotopic pregnancy can be managed medically under the following conditions: early and accurate diagnosis of the extra-uterine gestational sac and absence of symptoms. ${ }^{10}$ Several modalities have been reported with success: ultrasound-guided vaginal aspiration or in situ injection of methotrexate, potassium chloride, or hyperosmolar glucose. ${ }^{12}$ Expectant management of the ectopic pregnancy can be considered if it is not evolving. ${ }^{11}$

In present case three main measures were taken to preserve the intra-uterine pregnancy -short duration of general anaesthesia, minimal handling of the uterus during surgery and supplementation with progesterone. A literature review found survival rate of $64.4 \%$ for the intra-uterine foetus. Miscarriage can occur and its frequency in the aforementioned series was $35.6 \% .^{11}$

\section{CONCLUSION}

Heterotopic pregnancy can occur in the absence of any predisposing risk factors, and the detection of an intrauterine pregnancy does not exclude the possibility of the simultaneous existence of an ectopic pregnancy. Hence, in all patients of reproductive age, even in the presence of an intrauterine pregnancy, a complete review of the whole pelvis including adnexa should be done at the time of ultrasound to rule out the presence of a HP. $\mathrm{HP}$ although rare is a potentially life-threatening condition that, potentially has grave implications for both the mother and fetus. High-risk groups warrant early pregnancy ultrasound as a part of routine antenatal care to enable early diagnosis and timely management.

\section{ACKNOWLEDGMENTS}

Authors would like to thank teaching faculty of obstetrics and Gynecology department of Bangalore medical college for their whole-hearted support.

\section{Funding: No funding sources \\ Conflict of interest: None declared \\ Ethical approval: Not required}

\section{REFERENCES}

1. Govindarajan MJ, Rajan R. Heterotopic pregnancy in natural conception. J Hum Reprod Sci. 2008 Jan;1(1):37-38.

2. Hassani KI, Bouazzaoui AE, Khatouf M, Mazaz K Heterotopic pregnancy: A diagnosis we should suspect more often. J Emerg Trauma Shock. 2010;3:304.

3. Reece EA, Petrie RH, Sirmans MF, Finster M, Todd WD. Combined intrauterine and extrauterine gestations: a review. Am J Obstet Gynecol. 1983;146:323-30.

4. Devoe RW, Pratt JH. Simultaneous intrauterine and extrauterine pregnancy. Am J Obstet Gynecol. 1948;56:1119-26. 
5. McDonald J, Sebrina DP. Abdominal Pain In The Adolescent Female. Clin Pediatr Emerg Med. 2002;3:33-44.

6. Berger MJ, Taymor ML. Simultaneous intrauterine and tubal pregnancies following ovulation induction. Am J Obstet Gynecol. 1972;113:812-3.

7. Chen KH, Chen LR. Rupturing heterotopic pregnancy mimicking acute appendicitis. Taiwan $\mathrm{J}$ Obstet Gynecol. 2014;53:401-3.

8. Tal J, Haddad S, Gordon N, Timor-Tritsch I. Heterotopic pregnancy after ovulation induction and assisted reproductive technologies: a literature review from 1971 to 1993 . Fertil Steril. 1996;66:112.

9. Bright DA, Craupp FB. Heterotopic pregnancy: a reevaluation. J Am Board Fam Pract. 1990;3:125-8.

10. Fernandez H, Lelaidier C, Doumerc S, Fournet P, Olivennes F, Frydman R. Nonsurgical treatment of heterotopic pregnancy: a report of six cases. Fertil Steril. 1993;60(3):428-32.

11. Diallo D, Aubard Y, Piver P, Baudet JH. Grossesse hétérotopique: à propos de cinq cas et revue de la littérature. J Gynecol Obstet Biol Reproduction. 2000;29(2):131-14.

12. Allison JL, Aubuchon M, Leasure JD, Schust DJ. Hyperosmolar glucose injection for the treatment of heterotopic ovarian pregnancy. Obstet Gynecol. 2012;120(2):449-52.

Cite this article as: Spandana JC, Kanakannavar SS, Umashankar KM, Manuja N. Spontaneous heterotopic pregnancy with tubal rupture. Int J Reprod Contracept Obstet Gynecol 2017;6:5148-51. 